J. YONG

KODAI MATH. J.

15 (1992), 193-220

\title{
EXISTENCE THEORY OF OPTIMAL CONTROLS FOR DISTRIBUTED PARAMETER SYSTEMS
}

\author{
BY JIONGMIN YONG
}

\begin{abstract}
A unified existence theory of optimal controls for general semilinear evolutionary distributed parameter systems is established under the framework of mild (or weak) solutions for evolution equations. The theory applies to optimal control problems with the state equations being parabolic, hyperbolic partial differential equations and ordinary retarded differential equations. The approach also applies to problems governed by elliptic partial differential equations as well as variational inequalities.
\end{abstract}

\section{$\S 1$. Introduction.}

In [2], an existence theory for the optimal controls of semilinear abstract evolutionary distributed parameter systems was established under the framework of strong solutions for evolution equations (see the references cited therein also). It was asked whether one can establish a similar theory under the framwork of mild (or weak) solutions for evolution equations. In [27], the author started to investigate such a problem for time optimal control problem of semilinear distributed parameter systems. The purpose of this paper is to continue the investigation and establish a unified existence theory of optimal controls for evolutionary semilinear distributed parameter systems under the framework of mild solutions for evolution equations. Also, the same approach applies to elliptic variational inequalities.

The essence of existence theory of optimal controls is to find conditions (as weak as possible), under which some minimizing sequence of admissible pairs is convergent in the set of all admissible pairs (then the existence of an optimal pair follows from the sort of lower semi-continuity of the cost functional). This convergence usually follows from three conditions: 1) the relative com-

* This work was partially supported by the Chinese NSF under Grant 1913050, the Chinese State Education Commission Science Foundation under Grant 9024617.

AMS(MOS) subject classifications. 49A22, 49A27

Keywords. distributed parameter system, existence of optimal control, Cesari property, mild solution.

Received September 25, 1991. 
pactness of trajectories in some function spaces; 2) the existence of measurable selections for some multifunctions; and 3 ) the convexity condition on some relevant set-valued functions. In finite dimensional case, 1) and 2) follow from some (very weak) conditions $([5,14])$. Thus, essentially, the only condition imposed is the convexity condition, which seems to be indispensible in general. In the case that the control domain depending on the state, the convexity condition is given in terms of the so-called Cesari property (or the property $(Q)[5,10,14])$. In infinite dimensional case, to obtain a similar theory, the convexity condition still has to be imposed, of course. Again, in the case that the control domain depending on the state, such a convexity condition is imposed in terms of the Cesari property. On the other hand, some relevant measurable selection theorems for multifunctions valued in general (separable) metric spaces (including infinite dimensional separable Banach spaces) are still available $([16,26])$. Thus, the only thing left is the relative compactness of the trajectories in some functions spaces. This issue should be treated very carefully due to the infinite dimensionality nature of the space. It is very important to know that in using the Cesari property, we need the strong convergence of the trajectories. To archieve this, we have to impose sort of compactness conditions, one way or another. It is important that these conditions should be satisfied by the problems governed by usual concrete distributed parameter systems, say elliptic, parabolic, hyperbolic partial differential equations and retarded ordinary differential equations, etc. It turns out that our approarch not only covers the systems governed by mentioned equations, but also extends to some variational inequalities.

We should note that the problem studied in [6] basically is of elliptic partial differential system type because the highest order spatial derivative is solved explicitly. Also, the solution to the state equation was understood as a strong solution. It seems to us that there may be certain overlaps of our results with those in [12]. But, for the evolution problems, we use the semigroup and mild solution approach which is different from those in [12]; and for stationary problem, we discuss variational inequalities which was not mentioned in [12]. Recently, in $[19,21]$, also the strong solution was adopted in studying the similar problem for nonlinear evolution systems. For some other relevant results, we refer the readers to $[1,4,10,11,13,15,17,18,20,24,26]$.

\section{§2. Evolution Systems with Compact Semigroups.}

In this section, we establish the existence of optimal controls for semilinear evolutionary distributed parameter systems in which the semigroup involved is compact. This is the case for systems governed by semilinear parabolic partial differential equations with proper boudary conditions or by ratarded ordinary differential equations. 


\section{§ 2.1. Statement of the Control Problem.}

Let us start with the following assumptions.

(P1) $X$ is a reflexive Banach space, $U$ is a Polish space ([8]) with metric $d$, and $T>0$ is a constant.

(P2) Operator $A: \mathscr{D}(A) \subset X \rightarrow X$ generates a compact semigroup $e^{A t}$ on $X$.

(P3) Mapping $f:[0, T] \times X \times U \rightarrow X$ is Borel measurable in $(t, x, u)$ and continuous in $(x, u)$ for almost all $t \in[0, T]$; Mapping $f^{0}:[0, T] \times X \times U \rightarrow \boldsymbol{R}$ is Borel measurable in $(t, x, u)$, lower semicontinuous in $(x, u)$ and there exists a constant $K \geqq 0$, such that

$$
f^{0}(t, x, u) \geqq-K, \quad \forall(t, x, u) \in[0, T] \times X \times U .
$$

(P4) Multifunction $\Gamma:[0, T] \times X \rightarrow 2^{U}$ is pseuedo-continuous (see Appendix for definitions and relevant results about multifunctions).

(P5) $Q \subset X$ is closed and $\Omega \subset X \times X$ is bounded and weakly closed.

Next, we let

$$
v[0, T]=\{u:[0, T] \rightarrow U \mid u(\cdot) \text { is measurable }\} .
$$

Any element in $\mathcal{U}[0, T]$ is called a control (on $[0, T]$ ). The evolution system we are interested in is the following

$$
\dot{x}(t)=A x(t)+f(t, x(t), u(t)), \quad t \in[0, T] .
$$

As usual, a (mild) solution $x(\cdot)$ of $(2.2)$ is defined as a solution of the following integral equation:

$$
x(t)=e^{A t} x(0)+\int_{0}^{t} e^{A(t-\tau)} f(\tau, x(\tau), u(\tau)) d \tau, \quad t \in[0, T] .
$$

Any solution $x(\cdot)$ of $(2.3)$ is referred as a trajectory of the evolution system corresponding to initial state $x(0)$ and control $u(\cdot)$.

DEFINITION 2.1. A pair $(x(\cdot), u(\cdot))$ is said to be semi-admissible if (2.3) is satisfied and

$$
u(t) \in \Gamma(t, x(t)), \quad \text { a.e. } t \in[0, T] .
$$

The pair is said to be admissible if it is semi-admissible and

$$
\begin{gathered}
(x(0), x(T)) \in \Omega, \\
x(t) \in Q \quad t \in[0, T], \\
f^{0}(\cdot, x(\cdot), u(\cdot)) \in L^{1}(0, T) .
\end{gathered}
$$

In the case $(x(\cdot), u(\cdot))$ is semi-admissible (admissible, resp.), we refer $x(\cdot), u(\cdot)$ and $(x(\cdot), u(\cdot))$ semi-admissible (admissible) trajectory, control and pair, respectively. Hereafter, we let 


$$
\begin{aligned}
& \mathcal{A}_{s}=\{(x(\cdot), u(\cdot)) \in C([0, T] ; X) \times \mathcal{U}[0, T] \mid(x(\cdot), u(\cdot)) \text { is semi-admissible }\}, \\
& \mathscr{X}_{s}=\left\{x(\cdot) \in C([0, T] ; X) \mid \exists u(\cdot) \in \mathcal{U}[0, T], \text { such that }(x(\cdot), u(\cdot)) \in \mathcal{A}_{s}\right\}, \\
& \left.\mathcal{U}_{s}=\{u(\cdot)) \in \mathcal{U}[0, T] \mid \exists x(\cdot) \in C([0, T] ; X), \text { such that }(x(\cdot), u(\cdot)) \in \mathcal{A}_{s}\right\} .
\end{aligned}
$$

We define $\mathcal{A}_{a d}, \mathscr{X}_{a d}$ and $\mathcal{V}_{a d}$ similar to $\mathcal{A}_{s}, \mathfrak{X}_{s}$ and $\mathcal{V}_{s}$ replacing semi-admissibility by admissibility. Next, we introduce the cost functional to be of the following Lagrange form:

$$
J(x(\cdot), u(\cdot))=\int_{0}^{T} f^{0}(t, x(t), u(t)) d t, \quad \forall(x(\cdot), u(\cdot)) \in \mathcal{A}_{a d} .
$$

Then, our optimal control problem can be stated as follows.

Problem P. Find $(\bar{x}(\cdot), \bar{u}(\cdot)) \in \mathcal{A}_{a d}$, such that

$$
J(\bar{x}(\cdot), \bar{u}(\cdot))=\min _{(x(\cdot), u(\cdot)) \in \mathcal{A}_{a} d} J(x(\cdot), u(\cdot)) .
$$

If such a pair $(\bar{x}(\cdot), \bar{u}(\cdot))$ exists, we call $\bar{x}(\cdot), \bar{u}(\cdot)$ and $(\bar{x}(\cdot), \bar{u}(\cdot))$ an optimal trajectory, control and pair, respectively.

For simplicity, in this paper, we only consider the fixed duration problem. Our approach also applies to the case with non-fixed duration problem (see [27] for time optimal control problem). We should note that unlike in the finite dimensional case, the non-fixed duration problem can not be simply transformed to a fixed duration one (as in [5]). Also, our approach applies to problems with Mayer or Bolza type cost functionals.

\section{$\S 2.2$. Cesari Property.}

Let us recall the following.

Definition 2.2. ( $[5,14])$ Let $Y$ be a Banach space and $Z$ be a metric space. Let $\Lambda: Z \rightarrow 2^{Y}$ be a multifunction. We say $\Lambda$ possesses the Cesari property at $z_{0} \in Z$, if

$$
\bigcap_{\delta>0} \overline{c o} \Lambda\left(\Re_{\delta}\left(z_{0}\right)\right)=\Lambda\left(z_{0}\right) \text {, }
$$

where $\overline{c o} D$ stands for the closed convex hull of the set $D$ and $\eta_{\delta}\left(z_{0}\right)$ is the $\delta$ open neighborhood of the point $z_{0}$. If $\Lambda$ has the Cesari property at every point $z \in Z$, we simply say that $\Lambda$ has the Cesari property on $Z$.

Proposition 2.3. Let $Y$ be a Banach space and $Z$ be a metric space. Let $\Lambda: Z \rightarrow 2^{Y}$ be upper semicontinuous, convex and closed valued. Then, $\Lambda$ has the Cesari property on $Z$.

Proof. Let $z_{0} \in Z$ be fixed. For any $\varepsilon>0$, there exists a $\delta>0$ such that

$$
\Lambda\left(\Re_{\delta}\left(z_{0}\right)\right) \subset \Re_{\varepsilon}\left(\Lambda\left(z_{0}\right)\right) \text {. }
$$


Since $\Lambda\left(z_{0}\right)$ is convex, so is $\Re_{\varepsilon}\left(\Lambda\left(z_{0}\right)\right)$. Thus,

$$
\overline{c o} \Lambda\left(\Re_{\delta}\left(z_{0}\right)\right) \subset \overline{\Re_{s}\left(\Lambda\left(z_{0}\right)\right)} \text {. }
$$

then, it follows that

$$
\bigcap_{\delta>0} \overline{c o} \Lambda\left(\Re_{\tilde{\delta}}\left(z_{0}\right)\right) \subset \bigcap_{\varepsilon>0} \overline{\Re_{\varepsilon}\left(\Lambda\left(z_{0}\right)\right)}=\overline{\Lambda\left(z_{0}\right)}=\Lambda\left(z_{0}\right),
$$

which proves our proposition.

Now, let us introduce the following set

$$
\begin{gathered}
\mathcal{E}(t, x)=\left\{\left(y^{0}, y\right) \in \boldsymbol{R} \times X \mid y^{0} \geqq f^{0}(t, x, u), y=f(t, x, u), u \in \Gamma(t, x)\right\}, \\
\forall(t, x) \in[0, T] \times X .
\end{gathered}
$$

In the proof of the existence of optimal pairs for Problem $\mathrm{P}$, the following hypothese will play a cruicial role.

(P6) For almost all $t \in[0, T]$, the map $\mathcal{E}(t, \cdot): X \rightarrow 2^{R \times X}$ has the Cesari property on $Q$.

Following result gives a sufficient condition ensuring (P6).

Proposition 2.4. Let the following hold.

$\left(\mathrm{P}^{\prime}\right)$ For almost all $t \in[0, T]$, the map $f(t, \cdot, \cdot)$ is contınuous uniformly in $u \in U$ and $f^{0}(t, \cdot, \cdot)$ is lower semicontınuous uniformly in $u \in U$, i.e., for any given $x \in X$ and any $\varepsilon>0$, there exists $a \sigma=\sigma(x)>0$, such that whenever $x^{\prime} \in$ $n_{\sigma}(x)$ and $d\left(u, u^{\prime}\right)<\sigma$, one has

$$
\left\{\begin{array}{l}
\left|f\left(t, x^{\prime}, u^{\prime}\right)-f(t, x, u)\right|<\varepsilon, \\
f^{0}\left(t, x^{\prime}, u^{\prime}\right)>f^{0}(t, x, u)-\varepsilon .
\end{array}\right.
$$

$\left(\mathrm{P}^{\prime}\right)$ For almost all $t \in[0, T]$, the map $\Gamma(t, \cdot): X \rightarrow 2^{U}$ is upper semicontinuous on $X$.

Then, for almost all $t \in[0, T], \mathcal{E}(t, \cdot)$ has the Cesari property on $X$ if and only if for almost all $t \in[0, T]$, the set $\mathcal{E}(t, x)$ is convex and closed.

Remark 2.5. The above proposition looks very similar to the result of $[5$, pp. 72-74] for finite dimensional situation. However, here, we are in infinite dimensional space and we have not assumed any compactness! In some sense, our result can be regarded as an improvement of the one just mentioned. We will see that the proof given below is different from that given in [5].

Proof of Proposition 2.4. Let $t \in[0, T]$ be given such that $\left(\mathrm{P}^{\prime}\right)$ and $\left(\mathrm{P}^{\prime}\right)$ hold at this $t$ and let $x \in X$ be fixed. By (P3'), for any $\varepsilon>0$, there exists a $\sigma=\sigma(x)>0$, such that whenever $x^{\prime} \in \Re_{\sigma}(x)$ and $d\left(u, u^{\prime}\right)<\sigma,(2.15)$ holds. Next, by $\left(\mathrm{P} 4^{\prime}\right)$, we can find $0<\delta=\delta(x)<\sigma$, such that 


$$
\Gamma\left(t, \Re_{\delta}(x)\right) \subset \Re_{\sigma}(\Gamma(t, x)) .
$$

Then, for any $\left(y_{0}^{\delta}, y^{\delta}\right) \in \mathcal{E}\left(t, \Re_{\delta}(x)\right)$, there exist an $x^{\delta} \in \Re_{\delta}(x)$ and a $u^{\delta} \in \Gamma\left(t, x^{\delta}\right)$ $\subset \Re_{\sigma}(\Gamma(t, x))$, such that

$$
\left\{\begin{array}{l}
y_{0}^{\delta} \geqq f^{0}\left(t, x^{\delta}, u^{\delta}\right), \\
y^{\delta}=f\left(t, x^{\delta}, u^{\delta}\right) .
\end{array}\right.
$$

Then, it is clear that there exists a $u \in \Gamma(t, x)$ with $d\left(u^{\delta}, u\right)<\sigma$. Thus, note the fact that $\delta \leqq \sigma$, we see from (2.15) and (2.17) that

$$
\left\{\begin{array}{l}
y_{0}^{\delta} \geqq f^{0}\left(t, x^{\delta}, u^{\delta}\right)>f^{0}(t, x, u)-\varepsilon, \\
\left|y^{\delta}-f(t, x, u)\right|=\left|f\left(t, x^{\delta}, u^{\delta}\right)-f(t, x, u)\right|<\varepsilon .
\end{array}\right.
$$

This means

$$
\left(y_{0}^{\delta}, y^{\delta}\right) \in \Re_{\varepsilon}(\mathcal{E}(t, x)) .
$$

Hence, the set $\mathcal{E}(t, \cdot)$ is upper semicontinuous. Then our conclusion follows from Proposition 2.3.

In the above result, we see that $\left(\mathrm{P} 3^{\prime}\right)$ and $\left(\mathrm{P} 4^{\prime}\right)$ are not very restrictive. Thus, (P6) essentially says that $\mathcal{E}(t, x)$ is convex and closed. Following result gives a sufficient condition for $\mathcal{E}(t, x)$ being convex.

Proposition 2.6. Let $(t, x) \in[0, T] \times X$ be fixed. Let $f(t, x, \Gamma(t, x))$ be convex and there exists a convex function $\varphi(\cdot ; t, x): X \rightarrow \boldsymbol{R}$, such that

$$
f^{\circ}(t, x, u)=\varphi(f(t, x, u) ; t, x), \quad \forall u \in \Gamma(t, x) .
$$

Then, $\mathcal{E}(t, x)$ is convex.

Proof. Let $\left(y_{i}^{0}, y_{2}\right) \in \mathcal{E}(t, x), i=1,2$, and $\lambda \in(0,1)$. We can find $u_{1}, u_{2} \in$ $\Gamma(t, x)$, such that

$$
\left\{\begin{array}{l}
y_{i}^{0} \geqq f^{0}\left(t, x, u_{i}\right), \\
y_{i}=f\left(t, x, u_{i}\right),
\end{array} \quad i=1,2 .\right.
$$

By the convexity of $f(t, x, \Gamma(t, x))$, there exists a $u_{3} \in \Gamma(t, x)$, such that

$$
\lambda y_{1}+(1-\lambda) y_{2} \equiv \lambda f\left(t, x, u_{1}\right)+(1-\lambda) f\left(t, x, u_{2}\right)=f\left(t, x, u_{3}\right) .
$$

By (2.20), we have

$$
\begin{aligned}
\lambda y_{1}^{0}+(1-\lambda) y_{2}^{0} & \geqq \lambda f^{0}\left(t, x, u_{1}\right)+(1-\lambda) f^{0}\left(t, x, u_{2}\right) \\
& =\lambda \varphi\left(f\left(t, x, u_{1}\right) ; t, x\right)+(1-\lambda) \varphi\left(f\left(t, x, u_{2}\right) ; t, x\right)
\end{aligned}
$$




$$
\begin{aligned}
& \geqq \varphi\left(\lambda f\left(t, x, u_{1}\right)+(1-\lambda) f\left(t, x, u_{2}\right) ; t, x\right) \\
& =\varphi\left(f\left(t, x, u_{3}\right) ; t, x\right) \\
& =f^{0}\left(t, x, u_{3}\right) .
\end{aligned}
$$

Hence, $\mathcal{E}(t, x)$ is convex.

It is easy to check that if $f(t, x, u)$ is linear in $u, f^{0}(t, x, u)$ is convex in $u$ and $\Gamma(t, x)$ is convex, then $\mathcal{E}(t, x)$ is convex. The above result gives some other situations which guaranteeing the convexity of $\mathcal{E}(t, x)$. By some simple observation, we can see that in order the set $\mathcal{E}(t, x)$ to be convex, it is necessary that $f(t, x, \Gamma(t, x))$ is convex and the functions $f(t, x, \cdot), f^{0}(t, x, \cdot)$ and the set $\Gamma(t, x)$ should be compatible in some sense. Condition (2.20) is one of such compatibility conditions.

\section{$\S 2.3$. Properties of Compact Semigroups.}

In this subsection, we present a few results for compact $C_{0}$-semigroups. First of all, we have

Lemma 2.7. ([22]) Let $e^{A t}$ be a compact $C_{0}$-semigroup on Banach space $X$. Then, $t \mapsto e^{A t}$ is continuous in the operator norm in $(0, \infty)$.

Followig result will play an important role in sequel.

LEMMA 2.8. Let $e^{A t}$ be a compact $C_{0}$-semigroup on some Banach space $X$. Let $p>1$ and define

$$
\mathcal{S}(g(\cdot))=\int_{0}^{\cdot} e^{A(\cdot-\tau)} g(\tau) d \tau, \quad \forall g(\cdot) \Subset L^{p}(0, T ; X) .
$$

Then, $\mathcal{S}: L^{p}(0, T ; X) \rightarrow C([0, T] ; X)$ is compact.

It seems to us that such a result should be known. But we could not find an exact reference. For reader's convenience, we present a proof in the following.

Proof of Lemma 2.6. Let $g_{k}(\cdot) \in L^{p}(0, T ; X)$ with

$$
\left\|g_{k}(\cdot)\right\|_{L^{p}(0, T ; X)} \leqq 1, \quad \forall k \geqq 1 .
$$

We need to prove that $\left\{S\left(g_{k}(\cdot)\right)\right\}_{k \geqq 1}$ is relatively compact in $C([0, T] ; X)$. To this end, we first prove that for each $t \in[0, T]$, the set $\left\{\mathcal{S}\left(g_{k}(\cdot)\right)(t)\right\}_{k \geqq 1}$ is relatively compact in $X$. In fact, the case $t=0$ is trivial. We let $t \in(0, T]$. Then, for any $\varepsilon>0$, there exists a $\delta \in(0, t]$, such that

$$
\left|\int_{t-\delta}^{t} e^{A(t-r)} g_{k}(\tau) d \tau\right|<\frac{\varepsilon}{2}, \quad \forall k \geqq 1
$$

Next, we let 


$$
y_{k} \equiv \int_{0}^{t-\delta} e^{A(t-\delta-\tau)} g_{k}(\tau) d \tau, \quad \forall k \geqq 1 .
$$

Then, we see that the set $\left\{y_{k}\right\}_{k \geq 1}$ is bounded in $X$. Thus by the compactness of $e^{A \delta}$, we can find a finite set $\left\{z_{\imath}, 1 \leqq i \leqq m\right\}$ in $X$, such that

$$
\left\{e^{A \delta} y_{k}\right\}_{k \geqq 1} \subset \bigcup_{\imath=1}^{m} \Re_{\varepsilon / 2}\left(z_{\imath}\right) .
$$

Then, we have

$$
\left\{\mathcal{S}\left(g_{k}(\cdot)\right)(t)\right\}_{k \geq 1} \subset \bigcup_{\imath=1}^{m} \Re_{\varepsilon}\left(z_{\imath}\right) .
$$

Hence, for each $t \in[0, T],\left\{S\left(g_{k}(\cdot)\right)(t)\right\}_{k_{21} 1}$ is relatively compact in $X$. Next, we show that $\left\{S\left(g_{k}(\cdot)\right)\right\}_{k \geqslant 1}$ is equicontinuous on $[0, T]$. In fact, for $t^{\prime}>t>0$ and $0<\delta \leqq t$, we have

$$
\begin{aligned}
\mathcal{S}\left(g_{k}(\cdot)\right)\left(t^{\prime}\right)-\mathcal{S}\left(g_{k}(\cdot)\right)(t)=\int_{t}^{t^{\prime}} e^{A\left(t^{\prime}-\tau\right)} g_{k}(\tau) d \tau \\
\quad+\int_{0}^{t-\delta}\left(e^{A\left(t^{\prime}-\tau\right)}-e^{A(t-\tau)}\right) g_{k}(\tau) d \tau \\
\quad+\int_{t-\delta}^{t}\left(e^{A\left(t^{\prime}-\tau\right)}-e^{A(t-\tau)}\right) g_{k}(\tau) d \tau \\
\equiv I_{1}+I_{2}+I_{3} .
\end{aligned}
$$

By some direct estimation, we can find some constant $C$, independent of $k$. such that

$$
\begin{aligned}
& \left|I_{1}\right| \leqq C\left|t^{\prime}-t\right|^{p / p-1}, \\
& \left|I_{2}\right| \leqq C\left(\int_{\delta}^{t}\left\|e^{A\left(t^{\prime}-t+\tau\right)}-e^{A \tau}\right\|^{q} d \tau\right)^{1 / q} \\
& \left|I_{3}\right| \leqq C \delta^{1 / q}
\end{aligned}
$$

with $1 / p+1 / q=1$. Thus, by Lemma 2.7 , we obtain the equicontinuity of the set $\left\{\mathcal{S}\left(g_{k}(\cdot)\right)\right\}$ on $[0, T]$. Then by Arzela-Ascoli Theorem ([28]), we obtain the compactness of the operator $\mathcal{S}$.

COROLlary 2.9. Let $e^{A t}$ be a compact $C_{0^{-}}$-semigroup on some Banach space $X$ and $p>1$. Let $g_{k}(\cdot) \in L^{p}(0, T ; X)$ satisfy

$$
g_{k}(\cdot) \stackrel{w}{\longrightarrow} g(\cdot), \quad \text { in } \quad L^{p}(0, T ; X) .
$$

Then,

$$
\lim _{k \rightarrow \infty} \sup _{0 \leqq t \leqq T}\left\|\int_{0}^{t} e^{A(t-\tau)}\left(g_{k}(\tau)-g(\tau)\right) d \tau\right\|=0
$$




\section{§2.4. Existence of Semi-admissible Pairs.}

In this subsetion, we study the existence of semi-admissible pairs defined as in Definition 2.1. To this end, we first introduce the following:

(P3") The map $f:[0, T] \times X \times U \rightarrow X$ is Borel measurable in $(t, x, u)$, continuous in $(x, u)$ and for some constant $L>0$

$$
\left\{\begin{array}{l}
|f(t, x, u)-f(t, \hat{x}, u)| \leqq L|x-\hat{x}|, \quad \forall t \in[0, \infty), x, \hat{x} \in X, u \in U, \\
|f(t, x, u)| \leqq L(1+|x|), \quad \forall t \in[0, \infty), x \in X, u \in U .
\end{array}\right.
$$

$\left(\mathrm{P}^{\prime}\right)$ For almost all $t \in[0, \infty)$, the set $f(t, x, \Gamma(t, x))$ satisfies the following :

$$
\bigcap_{\delta>0} \overline{c o} f\left(t, \Re_{\delta}(x), \Gamma\left(\Re_{\delta}(t, x)\right)\right)=f(t, x, \Gamma(t, x)) .
$$

$\left(\mathrm{P}^{\prime \prime}\right)$ Let $\Lambda(t, x)=f(t, x, \Gamma(t, x))$. For almost all $t \in[0, T]$, the map $\Lambda(t, \cdot): X \rightarrow 2^{X}$ has the Cesari property.

It is clear that $\left(\mathrm{P}^{\prime}\right)$ implies $\left(\mathrm{P} 6^{\prime \prime}\right)$. In fact, one has

$$
\Lambda\left(t, \Re_{\delta}(x)\right) \subseteq f\left(t, \Re_{\delta}(x), \Gamma\left(\Re_{\delta}(t, x)\right)\right) .
$$

Furthermore, we have the following

Proposition 2.10. Let $\Gamma:[0, T] \times X \rightarrow 2^{U}$ be upper semicontinuous and closed set valued (see Appendix), $f(t, x, u)$ be uniformly continuous in $(x, u)$ for any $t \in[0, T]$. Then, the following are equivalent

(i) $\left(\mathrm{P} 6^{\prime}\right)$ holds.

(ii) (P6") holds.

(iii) $f(t, x, \Gamma(t, x))$ is closed and convex.

Proof. (i) $\Rightarrow$ (ii) $\Rightarrow$ (iii) are immediate.

(iii) $\Rightarrow($ i $)$. By the uniform continuity of $f(t, x, u)$ in $(x, u)$, for any $\varepsilon>0$, there exists a $\sigma>0$, such that

$$
f\left(t, \Re_{\sigma}(x), \Re_{\sigma}(\Gamma(t, x))\right) \subset \Re_{\varepsilon}(f(t, x, \Gamma(t, x))) .
$$

On the other hand, by the upper semicontinuity of $\Gamma$, we can find a $\delta \in(0, \sigma]$, such that

$$
\Gamma\left(\Re_{\delta}(t, x)\right) \subset \Re_{\sigma}(\Gamma(t, x)) .
$$

Thus, (i) follows from the convexity and closedness of $f(t, x, \Gamma(t, x))$.

Our main result of this subsection is the following:

Theorem 2.11. Let (P1), (P2), (P3'), (P4) and (P6') hold. Then, for any $y \in X$,

$$
\mathcal{A}_{s}([0, T] ; y) \equiv\left\{(x(\cdot), u(\cdot)) \in \mathcal{A}_{s} \mid x(0)=y\right\} \neq \varnothing .
$$


Moreover, $\mathfrak{X}_{s}([0, T] ; y) \equiv\left\{x(\cdot) \in \mathfrak{X}_{s} \mid x(0)=y\right\}$ is compact in $C([0, T] ; X)$.

Proof. For any $k \geqq 1$, Let

$$
t_{j}=\frac{j}{k} T, \quad 0 \leqq j \leqq k-1 .
$$

We set

$$
u_{k}(t)=\sum_{j=0}^{k-1} u^{j} \chi_{\left[t_{j}, t_{j+1}\right)}(t), \quad t \in[0, T] .
$$

Here, $u^{\jmath}$ 's are constructed as follows: First, we take

$$
u^{0} \in \Gamma(0, y) \text {. }
$$

By (2.29), we know that there exists a unique $x_{k}(\cdot)$ satisfies

$$
x_{k}(t)=e^{A t} y+\int_{0}^{t} e^{A(t-\tau)} f\left(\tau, x_{k}(\tau), u^{0}\right) d \tau, \quad \forall t \in\left[t_{0}, t_{1}\right] .
$$

Then, we take

$$
u^{1} \in \Gamma\left(t_{1}, x_{k}\left(t_{1}\right)\right)
$$

We can continue the above procedure to obtain $x_{k}(\cdot)$ on $\left[t_{1}, t_{2}\right]$, etc. By induction, we end up with the following:

$$
\left\{\begin{array}{l}
x_{k}(t)=e^{A t} y+\int_{0}^{t} e^{A(t-\tau)} f\left(\tau, x_{k}(\tau), u_{k}(\tau)\right) d \tau, \quad \forall t \in[0, T], \\
u_{k}(t) \in \Gamma\left(t_{\jmath}, x_{k}\left(t_{j}\right)\right), \quad t \in\left[t_{\jmath}, t_{\jmath+1}\right), \quad 0 \leqq j \leqq k-1 .
\end{array}\right.
$$

By Gronwall's inequality and (2.29), we see that for some absolute constant $C>0$.

$$
\left\{\begin{array}{l}
\left|x_{k}(t)\right| \leqq C, \quad \forall t \in[0, T], \\
\left|f\left(t, x_{k}(t), u_{k}(t)\right)\right| \leqq C, \quad \text { a.e. } t \in[0, T], \quad k \geqq 1 .
\end{array}\right.
$$

By Lemma 2.8, we know that $\left\{x_{k}(\cdot)\right\}_{k \geq 1}$ is relatively compact in $C([0, T] ; X)$. Then we may assume

$$
x_{k}(\cdot) \stackrel{s}{\longrightarrow} \bar{x}(\cdot), \quad \text { in } \quad C([0, T] ; X),
$$

for some $\bar{x}(\cdot) \in C([0, T] ; X)$. Also, we may let

$$
f\left(\cdot, x_{k}(\cdot), u_{k}(\cdot)\right) \stackrel{*}{\longrightarrow} \bar{f}(\cdot), \quad \text { in } \quad L^{\infty}(0, T ; X),
$$

for some $\vec{f}(\cdot) \in L^{\infty}(0, T ; X)$. By the compactness of the operator $\mathcal{S}$, we have

$$
\bar{x}(t)=e^{A t} y+\int_{0}^{t} e^{A(t-\tau)} \bar{f}(\tau) d \tau, \quad t \in[0, T] .
$$

By (2.38), for any $\delta>0$, there exists a $k_{0}$, such that 


$$
x_{k}(t) \in \Re_{\delta}(\bar{x}(t)), \quad \forall t \in[0, T], k \geqq k_{0} .
$$

On the other hand, by the definition of $u_{k}(\cdot)$, for $k$ large, one has

$$
u_{k}(t) \in \Gamma\left(t_{\jmath}, x_{k}\left(t_{j}\right)\right) \subset \Gamma\left(\Re_{\delta}(t, \bar{x}(t))\right), \quad \forall t \in\left[t_{\jmath}, t_{\jmath+1}\right), 0 \leqq j \leqq k-1 .
$$

Next, by (2.39) and Mazur Theorem, we may let $\alpha_{\imath \jmath} \geqq 0, \sum_{i \geqq 1} \alpha_{\imath \jmath}=1$, such that for some $p<\infty$,

$$
\phi_{j}(\cdot) \equiv \Sigma \alpha_{\imath \jmath} f\left(\cdot, x_{\imath+j}(\cdot), u_{\imath+j}(\cdot)\right) \stackrel{s}{\longrightarrow} \bar{f}(\cdot), \quad \text { in } \quad L^{p}(0, T, X) .
$$

Then, we can assume

$$
\phi_{\gamma}(t) \stackrel{s}{\longrightarrow} \bar{f}(t), \quad \text { in } X \text {, a.e. } t \in[0, T] .
$$

On the other hand, by (2.41) and (2.42), we see that for $j$ large, one has

$$
\phi_{j}(t) \in \operatorname{cof}\left(t, \Re_{\delta}(\bar{x}(t)), \Gamma\left(\Re_{\delta}(t, \bar{x}(t))\right)\right), \quad \text { a.e. } t \in[0, T] .
$$

Thus, for any $\delta>0$, we have

$$
\bar{f}(t) \in \overline{c o} f\left(t, \Re_{\delta}(\bar{x}(t)), \Gamma\left(\eta_{\delta}(t, \bar{x}(t))\right)\right), \quad \text { a.e. } t \in[0, T] .
$$

By $\left(\mathrm{P}^{\prime}\right)$, we get

$$
\bar{f}(t) \in f(t, \bar{x}(t), \Gamma(t, \bar{x}(t))), \quad \text { a.e. } \quad t \in[0, T] .
$$

Then, by Theorems A.3 and A.4 of the Appendix, we know that there exists a $\bar{u}(\cdot) \in \mathcal{U}[0, T]$, such that

$$
\left\{\begin{array}{l}
\bar{u}(t) \in \Gamma(t, \bar{x}(t)), \quad \text { a.e. } \quad t \in[0, T], \\
\bar{f}(t)=f(t, \bar{x}(t), \bar{u}(t)), \quad \text { a.e. } \quad t \in[0, T] .
\end{array}\right.
$$

Combining (2.40) and (2.48), we see that

$$
(\bar{x}(\cdot), \bar{u}(\cdot)) \in \mathcal{A}_{s}([0, T] ; y) .
$$

Thus, (2.34) follows. Finally, let $\left\{x^{k}(\cdot)\right\}_{k \geqq 1} \subset \mathfrak{X}_{s}([0, T] ; y)$ with

$$
\left\|x^{k}(\cdot)\right\|_{C([0, T] ; X)} \leqq C, \quad \forall k \geqq 1 .
$$

Then, by the above proof, we see that $\left\{x^{k}(\cdot)\right\}_{k \geq 1}$ is relatively compact in $C([0, T] ; X)$. Moreover, if for some subsequence (still denoted by itself), one has

$$
x^{k}(\cdot) \stackrel{s}{\longrightarrow} \hat{x}(\cdot), \quad \text { in } \quad C([0, T] ; X),
$$

then, by $\left(\mathrm{P} 6^{\prime}\right)$, we must have $\hat{x}(\cdot) \in \mathfrak{X}_{s}([0, T] ; y)$. Thus, $\mathfrak{X}_{s}([0, T] ; y)$ is compact in $C([0, T] ; X)$. 
We should note that in the case $\Gamma(t, x) \equiv \Gamma(t), \forall x \in X$, Theorem 2.11 holds under much simpler conditions and the proof becomes much simpler.

\section{$\S 2.5$. Existence of Optimal Pairs.}

In this subsetion, we present the existence of optimal pairs for Problem (P).

THEOREM 2.12. Let (P1)-(P6) hold. Let there exist a sequence of minimizing sequence $\left\{x_{k}(\cdot), u_{k}(\cdot)\right\} \in \mathcal{A}_{a d}$, such that for some $p>1$ and $C>0$,

$$
\left\|f\left(., x_{k}(\cdot), u_{k}(\cdot)\right)\right\|_{L^{p}(0, T ; X)} \leqq C, \quad \forall k \geqq 1 .
$$

Then, Problem $(P)$ admits at least one optimal pair.

Proof. Without loss of generality, we may assume

$$
f\left(\cdot, x_{k}(\cdot), u_{k}(\cdot)\right) \stackrel{w}{\longrightarrow} \bar{f}(\cdot), \quad \text { in } \quad L^{p}(0, T ; X),
$$

for some $\bar{f}(\cdot) \in L^{p}(0, T ; X)$. Then, by Corollary 2.9 , we obtain

$$
\begin{gathered}
\int_{0}^{t} e^{A(t-\tau)} f\left(\tau, x_{k}(\tau), u_{k}(\tau)\right) d \tau \stackrel{s}{\longrightarrow} \int_{0}^{t} e^{A(t-\tau)} \bar{f}(\tau) d \tau, \\
\text { uniformly in } t \in[0, T] .
\end{gathered}
$$

Also, by the boundedness of $\Omega$, refelxivity of $X$ and the compactness of $e^{A t}(t>0)$, we may assume

$$
\left\{\begin{array}{l}
x_{k}(0) \stackrel{w}{\longrightarrow} x_{0}, \\
e^{A t} x_{k}(0) \stackrel{s}{\longrightarrow} e^{A t} x_{0}, \quad \forall t \in(0, T] .
\end{array}\right.
$$

Let

$$
\bar{x}(t)=e^{A t} x_{0}+\int_{0}^{t} e^{A(t-\tau)} \bar{f}(\tau) d \tau, \quad t \in[0, T] .
$$

Then, by the above, we have

$$
\lim _{k \rightarrow \infty}\left\|x_{k}(t)-\bar{x}(t)\right\|=0, \quad t \in(0, T] .
$$

Then, by (2.50) and Mazur Theorem, we may let $\alpha_{\imath \jmath} \geqq 0, \Sigma_{i_{\gtrless 1}} \alpha_{i \jmath}=1$, such that for some $p<\infty$,

$$
\psi_{j}(\cdot) \equiv \sum_{i \geqq 1} \alpha_{i j} f\left(\cdot, x_{\imath+j}(\cdot), u_{i+j}(\cdot)\right) \stackrel{s}{\longrightarrow} \bar{f}(\cdot), \quad \text { in } \quad L^{p}(0, T ; X) .
$$

We set

$$
\phi_{j}^{0}(\cdot) \equiv \sum_{i \geqslant 1} \alpha_{i j} f^{0}\left(\cdot, x_{i+j}(\cdot), u_{i+j}(\cdot)\right)
$$

and set (note (2.1)) 


$$
\bar{f}^{0}(t)=\varliminf_{j \rightarrow \infty} \psi_{j}^{0}(t) \geqq-K, \quad \text { a.e. } \quad t \in[0, T] .
$$

Similar to [5], we have

$$
\left(\bar{f}^{0}(t), \bar{f}(t)\right) \in \mathcal{E}(t, \bar{x}(t)), \quad \text { a.e. } \quad t \in[0, T],
$$

and (by Fatou's Lemma)

$$
\int_{0}^{T} \bar{f}^{0}(t) d t \leqq \lim _{k \rightarrow \infty} J\left(x_{k}(\cdot), u_{k}(\cdot)\right)=\inf _{(x(\cdot), u(\cdot)) \in \mathcal{A} a d} J(x(\cdot), u(\cdot)) .
$$

Then, by Theorems A.3 and A.4 of the Appendix, we obtain $\bar{u}(\cdot)$, a measurable selection of $\Gamma(\cdot, \bar{x}(\cdot))$, such that

$$
\left\{\begin{array}{l}
\bar{f}^{0}(t) \geqq f^{0}(t, \bar{x}(t), \bar{u}(t)), \\
\bar{f}(t)=f(t, \bar{x}(t), \bar{u}(t)),
\end{array} \quad \text { a.e. } \quad t \in[0, T] .\right.
$$

Thus, noting (P5), we can easily see that $(\bar{x}(\cdot), \bar{u}(\cdot)) \in \mathcal{A}_{a d}$, and

$$
J(\bar{x}(\cdot), \bar{u}(\cdot)) \leqq \int_{0}^{T} \bar{f}^{0}(t) d t \leqq \inf _{(x(\cdot), u(\cdot)) \in \mathcal{A}_{a d}} J(x(\cdot), u(\cdot)) .
$$

This means that $(\bar{x}(\cdot), \bar{u}(\cdot))$ is an optimal pair.

We should note that (2.49) is a very weak condition. This condition holds if (P3") holds.

Remark 2.13. For the case $\Gamma(t, x) \equiv \Gamma(t), \forall x \in X$, we can relax the Cesari condition somehow (similar to [5]).

Remark 2.14, If the semigroup $e^{A t}$ is compact and analytic, then, the map $f$ can be more general. For example, in the case that $A$ is the Laplacian in some bounded domain in $\boldsymbol{R}^{n}$ with suitable boundary conditions, then, the nonlinear term $f$ is allowed to contain the first order spatial derivatives of the state. Of course, the assumptions ensuring the above results should be changed properly.

It is very easy to present concrete examples covered by the above theory. We prefer not to give these details here.

\section{§3. Second Order Evolution Systems.}

In this section, we will discuss the optimal control problems with the systems governed by following evolution equation:

$$
\ddot{x}(t)+A x(t)=f(t, x(t), u(t)), \quad t \in[0, T],
$$

with some symmetric operator $A$. The motivation is the controlled wave or beam equations. It is known that we may transfer the above into a first order 
evolution system. In fact, by setting (formally)

$$
z=\left(\begin{array}{c}
x \\
\dot{x}
\end{array}\right), \quad A=\left(\begin{array}{cc}
0 & I \\
-A & 0
\end{array}\right), \quad F(t, x, u)=\left(\begin{array}{c}
0 \\
f(t, x, u)
\end{array}\right),
$$

we have (formally again)

$$
\dot{z}(t)=\mathcal{A} z(t)+F(t, z(t), u(t)) .
$$

Then, it is standard that $\mathcal{A}$ generates a $C_{0}$-group (instead of a $C_{0}$-semigroup!) $e^{A t}$ in the underline space. Thus, $e^{A t}$ can not be compact! Then, the theory established in the previous section does not apply to the situation we have here. We note that in [2], the above type second order evolution controlled systems was not discussed.

\section{§3.1. Some Preliminary results on Second Order Evolution Systems.}

Let us start with some basic assumptions.

(W1) $V$ and $H$ are separable Hilbert spaces with norms $\|\cdot\|$ and $|\cdot|$, respectively, and with duals $V^{\prime}$ and $H^{\prime} \equiv H$, respectively. The embedding $V \hookrightarrow H$ is dense and compact. The duality pairing between $V$ and $V^{\prime}$ is $\langle\cdot, \cdot\rangle$ and the inner product of $H$ is $(\cdot, \cdot)$. The norm of $V^{\prime}$ is denoted by $\|\cdot\|_{*} \quad U$ is a Polish space ([8]) and $T$ is a positive constant.

(W2) $A \in \mathcal{L}\left(V, V^{\prime}\right)$ is symmetric and coercive, i.e.,

$$
\langle A x, y\rangle=\langle x, A y\rangle, \quad \forall x, y \in V,
$$

and for some $\alpha>0$,

$$
\langle A x, x\rangle \geqq \alpha\|x\|^{2}, \quad \forall x \in V .
$$

From (W1), we know that $V \hookrightarrow H=H^{\prime} \hookrightarrow V^{\prime}$. The space $H$ is usually referred as the pivot space. Now, for $g(\cdot) \in L^{2}\left(0, T ; V^{\prime}\right)$ and $\left(x_{0}, x_{1}\right) \in V \times H$, we consider the following evolution equation

$$
\ddot{x}(t)+A x(t)=g(t), \quad \text { a.e. } t \in[0, T] \text {, in } V^{\prime},
$$

with the initial conditions

$$
\begin{aligned}
& x(0)=x_{0}, \quad \text { in } H, \\
& \dot{x}(0)=x_{1}, \quad \text { in } V^{\prime} .
\end{aligned}
$$

We note that (3.6) and (3.7) stand for the following, respectively:

$$
\lim _{t \rightarrow 0}\left|x(t)-x_{0}\right|=0, \quad \lim _{t \rightarrow 0}\left\|\dot{x}(t)-x_{1}\right\|_{*}=0 .
$$

Before introducing the definition of a solution to (3.5)-(3.7), we first give the following result. 
Proposition 3.1. The following are equivalent.

(i) Function $x(\cdot) \in L^{2}(0, T ; V) \cap W^{1,2}([0, T] ; H) \cap W^{2,2}\left([0, T] ; V^{\prime}\right)$ satesfies (3.5)-(3.7).

(ii) Function $x(\cdot) \in L^{2}(0, T ; V) \cap W^{1,2}([0, T] ; H)$ satısfies (3.6) and

$$
\begin{gathered}
\int_{0}^{T}\langle g(t), v(t)\rangle d t=-\left(x_{1}, v(0)\right)-\int_{0}^{T}(\dot{x}(t), \dot{v}(t)) d t+\int_{0}^{T}\langle A x(t), v(t)\rangle d t, \\
\forall v(\cdot) \in L^{2}(0, T ; V) \cap W^{1,2}([0, T] ; H), \quad v(T)=0 \text { in } H .
\end{gathered}
$$

(iii) Function $x(\cdot) \in L^{2}(0, T ; V) \cap W^{1,2}([0, T] ; H)$ satisfies (3.6)-(3.7) and for any $v \in V,(\dot{x}(\cdot), v)$ is absolutely continuous in $[0, T]$ and

$$
\frac{d}{d t}(\dot{x}(t), v)+\langle A x(t), v\rangle=\langle g(t), v\rangle, \quad \text { a.e. } t \in[0, T] \text {. }
$$

The proof is straightforward. Then, we may give the following.

Definition 3.2. A function $x(\cdot) \in L^{2}(0, T ; V) \cap W^{1.2}([0, T] ; H)$ is called a solution of (3.5)-(3.7) if one of (i)-(iii) in Proposition 3.1 holds.

By usual Galerkin type method, we can prove the following result.

Proposition 3.3. Let $A$ satisfy (W2) and $\left(x_{0}, x_{1}\right) \in V \times H, g(\cdot) \in L^{1}(0, T ; H)$. Then, (3.5)-(3.7) admits a unique solution

$$
\begin{aligned}
x(\cdot) \in & L^{\infty}(0, T ; V) \cap W^{1, \infty}([0, T] ; H) \cap W^{2,2}\left([0, T] ; V^{\prime}\right) \\
& \hookrightarrow C([0, T] ; H) \cap C^{1}\left([0, T] ; V^{\prime}\right) .
\end{aligned}
$$

Moreover, it holds that

3.11, $|\dot{x}(t)|^{2}+\langle A x(t), x(t)\rangle \leqq\left\{\left[\left|x_{1}\right|^{2}+\left\langle A x_{0}, x_{0}\right\rangle\right]^{1 / 2}+\int_{0}^{t}|g(\tau)| d \tau\right\}^{2}, \quad t \in[0, T]$.

Next, we introduce another kind of solution, the so-called mild solution. To this end, we let

$$
\tilde{\mathcal{A}}=\left(\begin{array}{cc}
0 & 1 \\
-A & 0
\end{array}\right): V \times V^{\prime} \longrightarrow V^{\prime} \times V^{\prime},
$$

and let

$$
\left\{\begin{array}{l}
\mathscr{D}(\mathcal{A})=\{(x, y) \subseteq V \times H \mid \tilde{A}(x, y) \in V \times H\}=\{x \in V \mid A x \in H\} \times V, \\
\mathcal{A}=\tilde{A} \mid \mathscr{D}(\mathcal{A}): \mathscr{D}(\mathcal{A}) \subset V \times H \longrightarrow V \times H .
\end{array}\right.
$$

Following result is standard ([22]).

PRoposition 3.4. The operator $A$ generates a $C_{0}$ group on the space $V \times H$. If we let (formally) 


$$
z=\left(\begin{array}{l}
x \\
\dot{x}
\end{array}\right), \quad z_{0}=\left(\begin{array}{l}
x_{0} \\
x_{1}
\end{array}\right), \quad h=\left(\begin{array}{l}
0 \\
g
\end{array}\right)
$$

then, (3.5) reads

$$
\left\{\begin{array}{l}
\dot{z}(t)=\mathcal{A} z(t)+h(t), \\
z(0)=z_{0} .
\end{array}\right.
$$

Since $e^{A t}$ is a $C_{0}$-group on $Z \equiv V \times H$, we may study (3.14) directly without looking at (3.5). As usual, a function $z(\cdot) \in C([0, T] ; Z)$ is called a mild solution of (3.14) if it satisfies the following integral equation:

$$
z(t)=e^{\mathcal{A} t} z_{0}+\int_{0}^{t} e^{\mathcal{A}(t-\tau)} h(\tau) d \tau, \quad t \in[0, T] .
$$

On the other hand, adopted from [3], we introduce the notion of weak solution of (3.14) as follows:

Definition 3.5. A function $z(\cdot) \in C([0, T] ; Z)$ is called a weak solution of (3.14), if for any $z^{*} \in \mathscr{D}\left(\mathcal{A}^{*}\right)$, the map $\left\langle z(\cdot), z^{*}\right\rangle$ is absolutely continuous on $[0, T]$, and

$$
\left\{\begin{array}{l}
\frac{d}{d t}\left\langle z(t), z^{*}\right\rangle=\left\langle z(t), A^{*} z^{*}\right\rangle+\left\langle h(t), z^{*}\right\rangle, \quad \text { a.e. } t \in[0, T], \\
\left.\left\langle z(t), z^{*}\right\rangle\right|_{t=0}=\left\langle z_{0}, z^{*}\right\rangle .
\end{array}\right.
$$

It is important that we have the following equivalent result which is a consequence of the result of [3]. In the following lemma, $A$ and $Z$ need not to be the same as above.

LEMMA 3.6. Suppose $A$ generates a $C_{0}$-semigroup on some Banach space $Z$ and $h(\cdot) \in L^{1}(0, T ; Z)$. Then, $z(\cdot) \in C([0, T] ; Z)$ is a mild solution of (3.14) (i.e., (3.15) holds) if and only if $z(\cdot)$ is a weak solution of (3.14) (in the sense of Definition 3.5).

From this result, we end up with the following theorem.

TheOREM 3.7. Let (W2) hold and $g(\cdot) \in L^{1}(0, T ; H)$. Then,

(i) If $x(\cdot)$ is a solution of (3.5)-(3.7) in the sense of Definition 3.2, then, $\left(\begin{array}{l}x(\cdot) \\ \dot{x}(\cdot)\end{array}\right)$ is a mild solution of (3.14).

(ii) If $\left(\begin{array}{l}x(\cdot) \\ y(\cdot)\end{array}\right)$ is a mild solution of (3.14), then $y(\cdot)=\dot{x}(\cdot)$ and $x(\cdot)$ is a solution of (3.5)-(3.7) in the sense of Definition 3.2 .

Proof. First of all, we can easily check that 


$$
\left\{\begin{array}{l}
\mathscr{D}\left(\mathcal{A}^{*}\right)=H \times V, \\
\mathcal{A}^{*}\left(\begin{array}{l}
\varphi \\
\phi
\end{array}\right)=\left(\begin{array}{c}
-A \phi \\
\varphi
\end{array}\right) \equiv\left(\begin{array}{cc}
0 & -A \\
I & 0
\end{array}\right)\left(\begin{array}{l}
\varphi \\
\phi
\end{array}\right), \quad \forall\left(\begin{array}{l}
\varphi \\
\phi
\end{array}\right) \in \mathscr{D}\left(\mathcal{A}^{*}\right) .
\end{array}\right.
$$

(i) Let $x(\cdot)$ be a solution of (3.5)-(3.7) in the sense of Definition 3.2. Then, by setting $y(\cdot)=\dot{x}(\cdot)$, we have

$$
\frac{d}{d t}(y(t), \phi)=\langle-A x(t), \phi\rangle+(g(t), \phi), \quad \text { a.e. } \quad t \in[0, T], \quad \forall \psi \in V .
$$

It is easy to see that $\left(\right.$ since $\left.x(\cdot) \in W^{1,2}([0, T] ; H)\right)$

$$
\frac{d}{d t}(x(t), \varphi)=(y(t), \varphi), \quad \text { a.e. } \quad t \in[0, T], \quad \forall \varphi \in H .
$$

Thus, we have

$$
\begin{aligned}
\frac{d}{d t}\left\langle\left(\begin{array}{l}
x(t) \\
y(t)
\end{array}\right),\left(\begin{array}{l}
\varphi \\
\phi
\end{array}\right)\right\rangle= & (y(t), \varphi)+\langle-A x(t), \phi\rangle+(g(t), \phi) \\
= & \left\langle\left(\begin{array}{l}
x(t) \\
y(t)
\end{array}\right), A^{*}\left(\begin{array}{l}
\varphi \\
\phi
\end{array}\right)\right\rangle+\left\langle\left(\begin{array}{c}
0 \\
g(t)
\end{array}\right),\left(\begin{array}{l}
\varphi \\
\psi
\end{array}\right)\right\rangle, \\
& \forall\left(\begin{array}{l}
\varphi \\
\phi
\end{array}\right) \in H \times V \equiv \mathscr{D}\left(\mathcal{A}^{*}\right) .
\end{aligned}
$$

Thus, $\left(\begin{array}{l}x(\cdot) \\ y(\cdot)\end{array}\right)$ is a weak solution of (3.14). By Lemma 3.6, it is a mild solution of (3.14).

(ii) Let $\left(\begin{array}{l}x(\cdot) \\ y(\cdot)\end{array}\right)$ be a mild solution of (3.14). Then, it is a weak solution of (3.14) by Lemma 3.6. That means (3.18) and (3.19) hold. Then, it is ready to show that $x(\cdot) \in W^{1,2}([0, T] ; H)$ and

$$
y(\cdot)=\dot{x}(\cdot) \text {. }
$$

While, (3.19) is exactly the same as (3.9) and thus our conclusion follows.

Hereafter, we refer $x(\cdot)$ as a mild solution of (3.5)-(3.7) if $\left(\begin{array}{l}x(\cdot) \\ \dot{x}(\cdot)\end{array}\right)$ is a mild solution of (3.14). We have seen that the solutions defined in Definition 3.2 are mild solutions. We will simply refer them the solutions of (3.5)-(3.7).

\section{§ 3.2. Optimal Control Problem, Existence of Optimal Pairs.}

In this subsection, we first state our optimal control problem. Then, we will establish the existence of optimal pairs for our problem. First of all, we let Assumptions (W3), (W4) and (W6) be the same as (P3), (P4) and (P6), with $X$ being relpaced by $H$ and we also assume

(W5) The set $Q \subset H \times V^{\prime}$ is closed and $\Omega \subset V \times V \times H \times H$ is bounded and 
weakly closed.

Our controlled evolution system is given by (3.1). For any given $x_{0} \in V$, $x_{1} \in H$ and $u(\cdot) \in \mathcal{V}[0, T]$, we may talk about the mild (or weak) solution of

$$
\left\{\begin{array}{l}
\ddot{x}(t)+A x(t)=f(t, x(t), u(t)), \quad \text { a.e. } t \in[0, T], \\
x(0)=x_{0}, \\
\dot{x}(0)=x_{1} .
\end{array}\right.
$$

More precisely, we have the following.

Definition 3.8. A function $x(\cdot) \in L^{2}(0, T ; V) \cap W^{1,2}([0, T] ; H)$ is called a mild solution of (3.21) (corresponding to $x_{0} \in V, x_{1} \in H$ and $u(\cdot) \in \mathcal{V}[0, T]$ ) if $f(\cdot, x(\cdot), u(\cdot)) \in L^{2}(0, T ; H)$ and $x(\cdot)$ is a mild solution of (3.5) with $g(\cdot)$ being $f(\cdot, x(\cdot), u(\cdot))$.

DEFINITION 3.9. A pair $(x(\cdot), u(\cdot)) \in\left(L^{2}(0, T ; V) \cap W^{1,2}([0, T] ; H)\right) \times$ $\mathcal{U}[0, T]$ is said to be semi-admissible if $x(\cdot)$ is a mild solution of (3.21) corresponding to $u(\cdot)$ and

$$
u(t) \equiv \Gamma(t, x(t)), \quad \text { a.e. } t \in[0, T] .
$$

Moreover, if the following also hold:

$$
\begin{gathered}
(x(0), x(T), \dot{x}(0), \dot{x}(T)) \in \Omega \subset V \times V \times H \times H, \\
(x(t), \dot{x}(t)) \in Q \subset H \times V^{\prime}, \quad \text { a.e. } t \in[0, T], \\
f^{0}(\cdot, x(\cdot), u(\cdot)) \in L^{1}(0, T),
\end{gathered}
$$

then, we call $(x(\cdot), u(\cdot))$ an admissible pair.

We let

$A_{s}=\{(x(\cdot), u(\cdot)) \in C([0, T] ; H) \times \mathcal{V}[0, T] \mid(x(\cdot), u(\cdot))$ is semi-admissible $\}$,

and

$\mathcal{A}_{a d}=\{(x(\cdot), u(\cdot)) \in C([0, T] ; H) \times \mathcal{U}[0, T] \mid(x(\cdot), u(\cdot))$ is admissible $\}$.

Next, we introduce the cost functional.

$$
J(x(\cdot), u(\cdot))=\int_{0}^{T} f^{0}(t, x(t), u(t)) d t, \quad \forall(x(\cdot), u(\cdot)) \in \mathcal{A}_{a d} .
$$

Our optimal control problem can be stated as follows:

Problem W. Find $(\bar{x}(\cdot), \vec{u}(\cdot)) \in \mathcal{A}_{a d}$, such that

$$
J(\bar{x}(\cdot), \bar{u}(\cdot))=\inf _{(x(\cdot), u(\cdot)) \in \mathcal{A}_{a d}} J(x(\cdot), u(\cdot)) .
$$


If such a pair exists, we refer $\bar{x}(\cdot), \bar{u}(\cdot)$ and $(\bar{x}(\cdot), \bar{u}(\cdot))$ an optimal trajectory, control and pair, respectively.

Our main result of this scetion is the following theorem.

THEOREM 3.10. Let (W1)-(W6) hold. Let there exist a minimizing sequence $\left.\left\{x_{k}(\cdot), u_{k}(\cdot)\right)\right\} \subset \mathcal{A}_{a d}$, such that for some constant $C^{\prime}>0$,

$$
\int_{0}^{T}\left|f\left(t, x_{k}(t), u_{k}(t)\right)\right|^{2} d t \leqq C, \quad \forall k \geqq 1 .
$$

Then, Problem $W$ admits at least one optimal pair.

Proof. For each $k \geqq 1$, from (3.4), (3.11) and the boundedness of $\Omega$, we have that

$$
\begin{aligned}
&\left|\dot{x}_{k}(t)\right|^{2}+\alpha\left\|x_{k}(t)\right\|^{2} \leqq\left\{\left[\left|\dot{x}_{k}(0)\right|^{2}\right.\right. \\
&\left.\left.+\left\langle A x_{k}(0), x_{k}(0)\right\rangle\right]^{1 / 2}+\int_{0}^{t}\left|f\left(t, x_{k}(t), u_{k}(t)\right)\right| d \tau\right\}^{2} \leqq C, \\
& \forall t \in[0, T], \quad k \geqq 1,
\end{aligned}
$$

for some constant $C$. Thus, we may assume that

$$
\begin{cases}\dot{x}_{k}(\cdot) \stackrel{*}{\longrightarrow} \dot{\bar{x}}(\cdot), & \text { in } \quad L^{\infty}(0, T ; H), \\ x_{k}(\cdot) \stackrel{*}{\longrightarrow} \bar{x}(\cdot), & \text { in } \quad L^{\infty}(0, T ; V) .\end{cases}
$$

Due to the compactness of the embedding $V \subset \rightarrow H$, we know that the embedding

$$
L^{2}(0, T ; V) \cap W^{1,2}([0, T] ; H) \hookrightarrow L^{2}(0, T ; H),
$$

is compact ([23]). Thus, we may let

$$
\begin{gathered}
x_{k}(\cdot) \stackrel{s}{\longrightarrow} \bar{x}(\cdot), \quad \text { in } \quad L^{2}(0, T ; H), \\
x_{k}(t) \stackrel{s}{\longrightarrow} \bar{x}(t), \quad \text { in } H, \quad \text { a.e. } t \in[0, T] .
\end{gathered}
$$

On the other hand, by the boundedness of $\Omega$, we may assume

$$
\begin{cases}x_{k}(0) \stackrel{w}{\longrightarrow} \bar{x}_{0}, & \text { in } V, \\ \dot{x}_{k}(0) \stackrel{w}{\longrightarrow} \bar{x}_{1}, & \text { in } H .\end{cases}
$$

Also, from (3.28), we may let

$$
f\left(\cdot, x_{k}(\cdot), u_{k}(\cdot)\right) \stackrel{w}{\longrightarrow} \bar{f}(\cdot), \quad \text { in } \quad L^{2}(0, T ; H) .
$$

Then, by Definition 3.2, for any $v(\cdot) \in L^{2}(0, T ; V) \cap W^{1,2}([0, T] ; H)$ with $v(T)$ $=0$, we have 


$$
\begin{gathered}
\int_{0}^{T}\left\langle f\left(t, x_{k}(t), u_{k}(t)\right), v(t)\right\rangle d t=-\left(\dot{x}_{k}(0), v(0)\right) \\
\quad-\int_{0}^{T}\left(\dot{x}_{k}(t), \dot{v}(t)\right) d t+\int_{0}^{T}\left\langle x_{k}(t), A v(t)\right\rangle d t .
\end{gathered}
$$

Let $k \rightarrow \infty$, we obtain

$$
\begin{aligned}
& \int_{0}^{T}\langle\bar{f}(t), v(t)\rangle d t=-\left(x_{1}, v(0)\right) \\
& \quad-\int_{0}^{T}(\bar{x}(t), \dot{v}(t)) d t+\int_{0}^{T}\langle\bar{x}(t), A v(t)\rangle d t .
\end{aligned}
$$

From (3.32), we can find $\sigma \in(0, T)$, such that

$$
x_{k}(\sigma) \stackrel{s}{\longrightarrow} \bar{x}(\sigma) \text {. }
$$

Then, for any $h \in H$, we have

$$
\begin{aligned}
\left(x_{0}, h\right) & =\lim _{k \rightarrow \infty}\left(x_{k}(0), h\right) \\
& =\lim _{k \rightarrow \infty}\left\{\left(x_{k}(\sigma), h\right)-\int_{0}^{\sigma}\left(\dot{x}_{k}(\tau), h\right) d \tau\right\} \\
& =(\bar{x}(\sigma), h)-\int_{0}^{\sigma}(\bar{x}(\tau), h) d \tau \\
& =(\bar{x}(0), h) .
\end{aligned}
$$

That is

$$
\bar{x}(0)=x_{0}, \quad \text { in } H .
$$

By Definition 3.2, we know that

$$
\left\{\begin{array}{l}
\bar{x}(t)+A \bar{x}(t)=\bar{f}(t), \quad \text { a.e. } t \in[0, T], \quad \text { in } V^{\prime}, \\
x(0)=x_{0}, \quad \text { in } H, \\
\dot{x}(0)=x_{1}, \quad \text { in } V^{\prime} .
\end{array}\right.
$$

Then, by a similar method used in section 2 , we can prove that for some $\bar{f}^{0}(\cdot) \Leftarrow L^{1}(0, T)$,

$$
\left(\bar{f}^{0}(t), \bar{f}(t)\right) \in \overline{\operatorname{co}} \mathcal{E}(t, \bar{x}(t)), \quad \text { a.e. } \quad t \in[0, T],
$$

and

$$
\int_{0}^{T} \tilde{f}^{0}(t) d t \leqq \inf _{\mathcal{A}_{a d}} J(x(\cdot), u(\cdot)) .
$$

Then, by Theorems $\mathrm{A} 3$ and $\mathrm{A} 4$ of Appendix, we can find some $\bar{u}(\cdot) \in \mathcal{v}[0, T]$, such that 


$$
\left\{\begin{array}{l}
\bar{f}^{0}(t) \geqq f^{0}(t, \bar{x}(t), \bar{u}(t)), \\
\bar{f}(t)=f(t, \bar{x}(t), \bar{u}(t)), \quad \text { a. e. } \quad t \in[0, T] . \\
\bar{u}(t) \in \Gamma(t, \bar{x}(t)),
\end{array}\right.
$$

That is $(\bar{x}(\cdot), \bar{u}(\cdot)) \in \mathcal{A}_{a d}$. Hence, $(\bar{x}(\cdot), \bar{u}(\cdot))$ is an optimal pair.

We see that the key point is the strong convergence of the trajectories, which is guaranteed by the suitable compactness conditions. We also note that one can obtain the existence of semi-admissible pairs as we presented in section 2. Since the idea is the same, we omit the details here.

\section{§4. Elliptic Variational Inequalities.}

In this section, we are going to discuss the existence of optimal controls for elliptic variational inequalities. Let us start with some assumptions.

(E1) $\Omega$ is a bounded region in $\boldsymbol{R}^{n}$ with a smooth boundary $\partial \Omega, U$ is a metric space.

(E2) $\beta$ is a maximal monotone graph in $\boldsymbol{R} \times \boldsymbol{R}, A$ is a second order uniformly elliptic differential operator of divergince form. More precisely,

$$
A y(x)=-\sum_{\imath, j=1}^{n} \frac{\partial}{\partial x_{j}}\left(a_{\imath j}(x) \frac{\partial y(x)}{\partial x_{\imath}}\right), \quad \forall y(\cdot) \in H^{1}(\Omega),
$$

with $a_{\imath j}(\cdot) \in C^{1}(\bar{\Omega})$ and for some $\alpha_{0}>0$,

$$
\sum_{\imath, j=1}^{n} a_{\imath j}(x) \xi_{i} \xi_{\jmath} \geqq \alpha_{0}|\xi|^{2}, \quad \forall \xi \in \boldsymbol{R}^{n}, \quad x \in \Omega .
$$

(E3) $f: \Omega \times \boldsymbol{R} \times U \rightarrow \boldsymbol{R}$ is Borel measurable in $(x, y, u) \in \Omega \times \boldsymbol{R} \times \boldsymbol{R}$ and continuous in $(y, u) \in \boldsymbol{R} \times U$ for almost all $x \in \Omega, f^{0}: \Omega \times \boldsymbol{R} \times U \rightarrow \boldsymbol{R}$ is Borel measurable in $(x, y, u) \in \Omega \times \boldsymbol{R} \times \boldsymbol{R}$, lower semicontinuous in $(y, u) \in \boldsymbol{R} \times U$ for almost all $x \in \Omega$ and there exists a constant $K \geqq 0$, such that

$$
f^{0}(x, y, u) \geqq-K, \quad(x, y, u) \in \Omega \times \boldsymbol{R} \times U .
$$

(E4) $\Gamma: \Omega \times \boldsymbol{R} \rightarrow 2^{U}$ is pseudo-continuous.

(E5) $Q \subset \boldsymbol{R}$ is closed.

Our controlled system is the following:

$$
\left\{\begin{array}{l}
A y(x)+\beta(y(x)) \ni f(x, y(x), u(x)), \quad \text { a.e. } x \in \Omega, \\
\left.y\right|_{\partial \Omega}=0 .
\end{array}\right.
$$

Let us state the following result which will help us to understand (4.4).

Proposition 4.1. Let (E1), (E2) and the following (E3') hold (E3') $f: \Omega \times \boldsymbol{R} \times U \rightarrow \boldsymbol{R}$ is measurable in $(x, y, u) \in \Omega \times \boldsymbol{R} \times U$ and there exists 
a constant $L>0$, such that

$$
\begin{gathered}
|f(x, 0, u)| \leqq L, \quad \forall(x, u) \in \Omega \times U, \\
0 \geqq[f(x, y, u)-f(x, \hat{y}, u)](y-\hat{y}) \geqq-L|y-\hat{y}|^{2}, \\
\forall(x, u) \in \Omega \times U, \quad y, \hat{y} \in \boldsymbol{R} .
\end{gathered}
$$

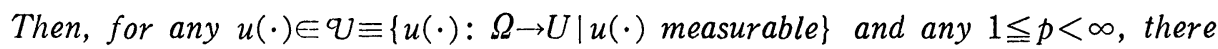
exists a unique solution $y(\cdot) \equiv y(\cdot ; u(\cdot)) \in W^{2, p}(\Omega) \cap H_{0}^{1}(\Omega)$ to (4.4). Moreover,

$$
|y(\cdot ; u(\cdot))|_{W^{2, p}(\Omega)} \leqq C_{p}, \quad \forall u(\cdot) \in \mathcal{U} .
$$

The proof can be carried out by using the method of [7] (see [9] also). Actually, from [7], we know that $\left(\mathrm{E} 3^{\prime}\right)$ can be more general if $U$ is a Banach space.

In our problem, the control and the state constraints are given by

$$
\begin{gathered}
u(x) \in \Gamma(x, y(x)), \quad \text { a.e. } \quad x \in \Omega, \\
y(x) \in Q, \quad \text { a.e. } \quad x \in \Omega .
\end{gathered}
$$

We let $p>n / 2$. A pair $(y(\cdot), u(\cdot)) \in\left(W^{2, p}(\Omega) \cap H_{0}^{1}(\Omega)\right) \times \mathcal{U}$ is said to be semiadmissible if (4.4) and (4.8) are satisfied and it is said to be admissible if (4.4), (4.8)-(4.9) hold and $f^{0}(\cdot, y(\cdot), u(\cdot)) \in L^{1}(\Omega)$. We use the notation $\mathcal{A}_{s}, \mathcal{A}_{a d}, \mathfrak{X}_{s}$, $\mathscr{X}_{a d}, \mathcal{V}_{s}$ and $\mathcal{V}_{a d}$ similar to those in $\S 2.1$. Next, for any $(y(\cdot), u(\cdot)) \in \mathcal{A}_{a d}$, we define the cost functional to be the following:

$$
J(y(\cdot), u(\cdot))=\int_{\Omega} f^{0}(x, y(x), u(x)) d x .
$$

Then, our optimal control problem can be stated as follows:

Problem E. Find $(\bar{y}(\cdot), \bar{u}(\cdot)) \in \mathcal{A}_{a d}$, such that

$$
J(\bar{y}(\cdot), \bar{u}(\cdot))=\inf _{A_{a d}} J(y(\cdot), u(\cdot)) .
$$

If a pair $(\bar{y}(\cdot), \bar{u}(\cdot)) \in \mathcal{A}_{a d}$ exists satisfying $(4.11)$, we call $(\bar{y}(\cdot), \bar{u}(\cdot)), \bar{y}(\cdot)$ and $\bar{u}(\cdot)$ an optimal pair, state and control, respectively.

The main purpose of this section is to establish the existence of an optimal pair of Problem E. To this end, let us first introduce the following set:

$$
\begin{gathered}
\mathcal{E}(x, y)=\left\{\left(z^{0}, z\right) \in \boldsymbol{R}^{2} \mid z^{0} \geqq f^{0}(x, y, u), z=f(x, y, u), u \in \Gamma(x, y)\right\}, \\
\forall(x, y) \in \Omega \times \boldsymbol{R} .
\end{gathered}
$$

We further introduce the following assumption (compare (P6) and (W6))

(E6) For almost all $x \in \Omega$, the map $y \mapsto \mathcal{E}(x, y)$ has the Cesari property on $Q$.

Now, we are ready to state and prove the main result of this section. 
Theorem 4.2. Let (E1)-(E6) hold. Let $\left.\left\{y_{k}(\cdot), u_{k}(\cdot)\right)\right\} \subset \mathcal{A}_{a d}$ be a minimizing sequence of Problem $E$, such that for some absolute constant $C$,

$$
\left|f\left(\cdot, y_{k}(\cdot), u_{k}(\cdot)\right)\right|_{L^{\infty}(\Omega)} \leqq C, \quad \forall k \geqq 1 .
$$

Then Problem $E$ aamits at teast one optimal pair.

Proof. From the idea of [7], we know that for $p>n / 2$,

$$
\left|y_{k}(\cdot)\right|_{W^{2, p}(\Omega)} \leqq C_{p}, \quad \forall k \geqq 1 .
$$

Thus, we may let

$$
\begin{array}{r}
y_{k}(\cdot) \longrightarrow \bar{y}(\cdot), \quad \text { weaky in } W^{2, p}(\Omega), \\
\text { strongly in } C^{\alpha}(\bar{\Omega}),
\end{array}
$$

for some $\alpha \in(0,1)$ and some $\bar{y}(\cdot) \in W^{2, p}(\Omega) \cap H_{0}^{1}(\Omega)$. On the other hand, we may let

$$
f\left(\cdot, y_{k}(\cdot), u_{k}(\cdot)\right) \longrightarrow \bar{f}(\cdot), \quad \text { weakly in } L^{p}(\Omega),
$$

for some $\bar{f}(\cdot) \in L^{\infty}(\Omega)$. Then, by Mazur Theorem, we can find $\alpha_{\imath \jmath} \geqq 0, \sum_{\imath: 1} \alpha_{\imath \jmath}$ $=1$, such that

(4.17) $\quad \phi_{j} \equiv \sum_{i \geqslant 1} \alpha_{\imath \jmath} f\left(\cdot, y_{\imath+j}(\cdot), u_{\imath+j}(\cdot)\right) \longrightarrow \bar{f}(\cdot), \quad$ strongly in $L^{p}(\Omega)$.

We set

$$
\phi_{j}^{0}(\cdot)=\sum_{i \geqq 1} \alpha_{\imath \jmath} f^{0}\left(\cdot, y_{\imath \jmath}(\cdot), u_{\imath+\jmath}(\cdot)\right),
$$

and let

$$
\bar{f}^{0}(x)=\varliminf_{k \rightarrow \infty} f^{0}\left(x, y_{k}(x), u_{k}(x)\right) \geqq-K, \quad \text { a.e. } \quad x \in \Omega .
$$

By (4.15), we see that for any $\varepsilon>0$, there exists a $\jmath_{0}$, such that for $j \geqq j_{0}$,

$$
\left(\psi_{j}^{0}(x), \phi_{i}(x)\right) \in \operatorname{coE}\left(x, \Re_{\varepsilon}(\bar{y}(x))\right), \quad \text { a. e. } \quad x \in \Omega .
$$

Thus,

$$
\left(\bar{f}^{0}(x), \bar{f}(x)\right) \in \overline{c o} \mathcal{E}\left(x, \Re_{\varepsilon}(\bar{y}(x))\right), \quad \text { a. e. } \quad x \in \Omega .
$$

Then, by (E6), we see that

$$
\left(\bar{f}^{0}(x), \bar{f}(x)\right) \in \mathcal{E}(x, \bar{y}(x)), \quad \text { a.e. } \quad x \in \Omega .
$$

Hence, similar to the previous sections, we can find $\bar{u}(\cdot) \subseteq \vartheta$, such that 


$$
\left\{\begin{array}{l}
\bar{f}^{0}(x) \geqq f^{0}(x, \bar{y}(x), \bar{u}(x)), \\
\bar{f}(x)=f(x, \bar{y}(x), \bar{u}(x)), \quad \text { a.e. } \quad x \in \Omega . \\
\bar{u}(x) \in \Gamma(x, \bar{y}(x)),
\end{array}\right.
$$

On the other hand, from the admissibility of $\left(y_{k}(\cdot), u_{k}(\cdot)\right)$, the convergence (4.15) and (4.16) and (4.23), we have (see [4])

$$
\left\{\begin{array}{l}
A \bar{y}(x)+\beta(\bar{y}(x)) \ni f(x, \bar{y}(x), \bar{u}(x)), \quad \text { a.e. } \quad x \in \Omega, \\
\left.\bar{y}\right|_{\partial \Omega}=0 .
\end{array}\right.
$$

By (E5), we have

$$
\bar{y}(x) \in Q, \quad \text { a.e. } \quad x \in \Omega \text {. }
$$

Hence, $(\bar{y}(\cdot), \bar{u}(\cdot)) \in \mathcal{A}_{a d}$. By Fatou's Lemma, we see that

$$
\begin{aligned}
J(\bar{y}(\cdot), \bar{u}(\cdot)) & =\int_{\Omega} f^{0}(x, \bar{y}(x), \bar{u}(x)) d x \\
& \leqq \lim _{k \rightarrow \infty} J\left(y_{k}(\cdot), u_{k}(\cdot)\right) \\
& =\inf _{\mathcal{A}_{a d}} J(y(\cdot), u(\cdot)) .
\end{aligned}
$$

Thus, $(\bar{y}(\cdot), \bar{u}(\cdot))$ is an optimal pair.

Remark 4.3. In the above the operator $A$ is not necessarily of second order. Also, we see that if $\beta=0$, the (4.4) is reduced to a semilinear elliptic partial differential system with the leading operator being of divergence form and coercive. Thus, our theory covers the existence of optimal controls for the mentioned elliptic systems.

Remark 4.4. From the above, we see that the existence of an optimal pair follows essentially from the precompactness of the minimizing sequence in suitable spaces and the sort of convexity conditions. The multivaluedness of term $A y+\beta(y)$ does not bring any difficulty. However, if this multivalued operator also depends on the control variable, then, the situation becomes technically difficult.

Remark 4.5. It seems to us that our approach is also possible to discuss the existence of optimal controls for evolutionary variational inequalities.

\section{Appendix. Multivalued Mappings.}

In this appendix, we recall some results about multivalued mappings. First, we recall the following definition.

Definition A.1. Let $T$ and $Z$ be metric spaces, $\Lambda: T \rightarrow 2^{Z} \equiv\{$ nonempty 
subsets of $Z$ \}.

(i) $\Lambda$ is said to be continuous (with respect to the Hausdorff metric $\rho_{H}$ ) at $t \in T$, if

$$
\lim _{\bar{t} \rightarrow t} \rho_{H}(\Lambda(t), \Lambda(t))=0,
$$

where

$$
\rho_{H}\left(\Lambda_{1}, \Lambda_{2}\right)=\frac{1}{2} \max \left\{\sup _{\lambda_{1} \in \Lambda_{1}} d\left(\lambda_{1}, \Lambda_{2}\right)+\sup _{\lambda_{2} \in \Lambda_{2}} d\left(\Lambda_{1}, \lambda_{2}\right)\right\} .
$$

(ii) $\Lambda$ is said to be lower semi-continuous at $t \in T$, if for any open set $V$, whenever

$$
\Lambda(t) \cap V \neq \varnothing
$$

there exists a $\delta>0$, such that

$$
\Lambda(s) \cap V \neq \varnothing, \quad \forall s \in \Re_{\delta}(t) .
$$

(iii) $\Lambda$ is said to be upper semi-continuous at $t \in T$, if for any $\varepsilon>0$, there exists a $\delta>0$, such that

$$
\Lambda\left(\Re_{\delta}(t)\right) \subset \Re_{\varepsilon}(\Lambda(t)) .
$$

(iv) $\Lambda$ is said to be pseudo-continuous at $t \in T$, if

$$
\bigcap_{\varepsilon>0} \overline{\Lambda\left(\mathscr{N}_{\varepsilon}(t)\right)}=\Lambda(t) \text {. }
$$

(v) If $T$ is a domain in $\boldsymbol{R}^{n}$, then, $\Lambda$ is said to be measurable, if for any closed subset $F \subset Z$, the set

$$
\Lambda^{-1} \equiv\{t \in T \mid \Lambda(t) \cap F \neq \varnothing\}
$$

is (Lebesgue) measurable.

(vi) $\Lambda$ is said to be closed and/or convex set valued on $T$ if for all $t \in T$, $\Lambda(t)$ is closed and/or convex.

If in (i )-(iv), the mentioned properties hold for all $t \in T$, we simply say $\Lambda$ is continuous, upper semi-continuous and psuedo-continuous, respectively.

Proposition A.2. Let $Z$ be a complete metric space and $\Lambda: T \rightarrow Z$ closed set valued. Then,

(i) If $T$ is a metric space, then, the following implication chains hold

$\Lambda$ is continuous $\Rightarrow \Lambda$ is lower semicontinuous

$\Longleftrightarrow \forall$ open set $F \subset Z, \Lambda^{-1}$ is open in $T$;

$\Lambda$ is continuous $\Rightarrow \Lambda$ is upper semicontinuous

$\Longleftrightarrow \forall$ closed set $F \subset Z, \Lambda^{-1}$ is closed in $T$ 


$$
\begin{aligned}
& \Rightarrow \Lambda \text { is psuedo-continuous } \\
& \Longleftrightarrow G(\Lambda) \equiv \text { the graph of } \Lambda \text { is closea in } T \times X .
\end{aligned}
$$

(ii) If $T$ is a metric space and $Z$ is a compact metruc space. Then $\Lambda$ is continuous $\Longleftrightarrow \Lambda$ is lower and upper semicontinuous;

$\Lambda$ is upper continuous $\Longleftrightarrow \Lambda$ is pseudo-continuous.

(iii) If $T$ is a domain in $\boldsymbol{R}^{n}$, then,

$\Lambda$ is psuedo-contınuous $\Rightarrow \Lambda$ is measurable.

In [5], the pseudo-continuity and the upper semicontinuity were referred as upper semicontinuity and upper semicontinuity with respect to the inclusion (u.s.c.i.), respectively. Our notion is adopted from [28] for lower, upper semicontinuity and a modification of that given in [2] for pseudo-continuity.

THEOREM A.3. ([16,25]) Let $T$ be a locally compact metric space, $U$ be a Polish space, $X$ be a complete metric space, $\Lambda: T \rightarrow 2^{U}$ be measurable taking closed subset values, $f: T \times U \rightarrow X$ be measurable in $t$, locally uniformly continuous in $x$ and $\bar{f}: T \rightarrow X$ be measurable with

$$
\bar{f}(t) \in f(t, \Lambda(t)), \quad \text { a.e. } \quad t \in T .
$$

Then, there exists a measurable function $u: T \rightarrow U$, such that

$$
\left\{\begin{array}{l}
u(t) \in \Lambda(t), \\
\vec{f}(t)=f(t, u(t)),
\end{array} \quad \text { a. e. } \quad t \in T .\right.
$$

Theorem A.4. Let $T$ be a domain in $\boldsymbol{R}^{n}, X$ be a metric space, $U$ be a Polish space, $\Gamma: T \times X \rightarrow 2^{U}$ be pseudo-continuous and $\bar{x}: T \rightarrow X$ be measurable. Then, $\Gamma(\cdot, \bar{x}(\cdot)): T \rightarrow 2^{U}$ is measurable.

The proof follows easily from the definition and the criteria of measurability for multifunctions given in [16].

\section{REFERENCES}

[1] N.U. Ahmed, Existence of optimal controls for a class of systems governed by differential inclusions on a Banach space, J. Optim. Theory \& Appl., 50 (1986), 213-237.

[2] N.U. Ahmed and K.L. Teo, Optimal Control of Distributed Parameter Systems, North Holland, New York, 1981.

[3] J.M. BALL, Strong continuous semigroups, weak solutions and the variation of constants formula, Proc. Amer. Soc., 63 (1972), 372-373.

[4] V. BARBU, Optimal Control of Variational Inequalities, Pitman, London, 1984. 
[5] L.D. Berkovitz, Optimal Control Theory, Springer-Verlag, New York, 1974.

[6] L.D. Berkovitz, Existence and lower closure theorems for abstract control problems, SIAM J. Control, 12 (1974), 27-42.

[7] J.F. Bonnans AND D. TiBA, Pontryagin principle in the control of semilinear elliptic variational inequalities, Appl. Math. Optim., 23 (1991), 299-312.

[8] N. Bourbaki, General Topology, Addison-Wesley Reading, 1966.

[9] H. Brezis, Problèmes unilatéreaux, J. Math. Pure Appl., 51 (1972), 1-168.

[10] L. CESARI, Existence theorems for weak and usual Lagrange problems with unilateral constraints I, II, Trans. Amer. Math. Soc., 124 (1966), 369-427.

[11] L. Cesari, Existence theorems for abstract multidimensional control problems, J. Optim. Theory \& Appl., 6 (1970), pp. 210-236.

[12] L. CESARI, Geometric and analytic views in existence theorems for optimal control in Banach spaces, I, II, III, J. Optim. Theory \& Appl., 14 (1974), 505520, 15 (1975), 467-497, 19 (1976), 185-214.

[13] L. CEsari, Existence of solutions and existence of optimal solutions, Mathematıcal Theories of Optımization, J.P. Cecconi and T. Zolezz1 eds., Lecture Notes in Math., Vol. 979, Springer-Verlag, Berlin, 1983, 88-107.

[14] L. Cesari, Optimization Theory and Applications, Problems with Ordinary Differential Equations, Sprınger-Verlag, New York, 1983.

[15] R.F. Curtain and A.J. Pritchard, Infinite Dimensional Linear Systems Theory, Lecture Notes in Control and Information Scieces, Vol. 8, Springer-Aerlag, New York, 1981.

[16] C.J. Himmelberg, M.Q. Jacobs and F.S. Van Vleck, Measurable multifunctions, selectors and Filippov's implicit functions lemma, J. Math. Anal. Appl., 25 (1969), 276-284.

[17] S.H. Hou, Existence theorems of optimal control problems in Banch spaces, Nonlinear Anal., 7 (1983), 239-257.

[18] S. Nababan And K.L. Teo, On the existence of optımal controls of the first boundary value problem for parabolic partial delay-differential equations in divergence form, J. Math. Soc. Japan, 32 (1980), 343-362.

[19] N.S. PAPAgEORgiou, Existence of optimal controls for nonlinear systems in Banach spaces, J. Optim. Theory \& Appl., 53 (1987), 451-459.

[20] N.S. PAPAgEorgiou, Properties of the relaxed trajectories of evolution equations and optimal controls, SIAM J. Control \& Optim. 27 (1989), 267-288.

[21] N.S. PAPAGEORgIOU, Existence of optimal controls for nonlinear distributed parameter systems, Funkcialaj Ekvacioj, 32 (1989), 429-437.

[22] A. PAZY, Semigroups of Linear Operators and Applications to Partial Differential Equations, Springer-Verlag, New York, 1983.

[23] R. Temam, Navier-Stokes Equations: Theory and Numerical Analysis, 3rd edition, North-Holland, Amsterdam, 1984.

[24] R. TORRE JÓN AND J. YONG, A control problem for clamped extensible beams, Appl. Anal., 28 (1988), 163-180.

[25] D.H. WAgner, Survey of measurable selection theorems, SIAM J. Control \& Optim., 15 (1977), 859-903.

[26] J. WARga, Optimal Control of Differential and Functional Equations, Academic Press, New York, 1972.

[27] J. Yong, Time optimal control for semilinear distributed parameter systemsexistence theory and necessary conditions, Kodai Math. J., 14 (1991), 239-253. 
[28] E. ZeIdLER, Nonlinear Functional Analysis and Its Applications, I, SpringerVerlag, New York, 1986.

Department of Mathematics,

FUDAN UNIVERSITY

SHANGHAI 200433,

CHINA 